\title{
Altersfreitod oder vernachlässigte Fürsorge
}

\author{
Hans Kurt \\ Dr. med., Past-president, Schweizerische Gesellschaft für Psychiatrie und Psychotherapie SGPP
}

\begin{abstract}
Vater war nicht lebensmüde, aber alterssatt (Ueli Oswald) - Viele wollen nicht zur Last fallen (Werner Schärer, Pro Senectute) - Gesundheitskosten für Rentner steigen um 4 Milliarden («Tages-Anzeiger») - Glücklich sterben heisst selbstbestimmt sterben (Hans Küng) - Tödliches Mitleid, zur sozialen Frage der Unerträglichkeit des Lebens (Klasus Dörner) - Britin stirbt in der Schweiz aus Angst, eine Greisin zu werden («Tages-Anzeiger») - Die letzte Reise von David Goodall, der 104-jährige Australier ist in die Schweiz gereist, um hier zu sterben - und einen Beitrag zur globalen Sterbehilfe-Debatte zu leisten.
\end{abstract}

\begin{abstract}
Schlagzeilen feuern die Diskussion um die Sterbehilfe an. Werbespots von Berühmtheiten aus Unterhaltung, Politik und Kultur tragen zur Debatte bei. Exit, die grösste Sterbehilfeorganisation in der Schweiz, zählt 110000 Mitglieder. Die Ärzteschaft gerät mit Slogans wie Behandlungswillkür oder gewinnbringende Krankheitsindustrie mit alten todkranken Menschen in den Brennpunkt des Diskurses. Wie so oft vermischen sich politische Forderungen mit ethischen Bedenken, und die Ärzte geraten in Gefahr, zu Ausführungsgehilfen für zum Teil missionarisch vertretene Ansichten zu werden.
\end{abstract}

\section{Bedingungen und Fragen zum Sterbewillen}

In den Richtlinien der Schweizerischen Akademie der medizinischen Wissenschaften SAMW aus dem Jahr 2013, die auch Teil der Standesordnung der FMH sind, wird festgehalten, dass Beihilfe zum Suizid nicht Teil

Es liegt in der persönlichen Entscheidung jedes Arztes, Beihilfe zum Suizid zu leisten oder eine solche abzulehnen.

der ärztlichen Tätigkeit ist, weil sie den Zielen der Medizin widerspricht. Es liegt in der persönlichen Entscheidung jedes Arztes, Beihilfe zum Suizid zu leisten oder eine solche abzulehnen. Der Arzt muss aber die Voraussetzungen zur Sterbehilfe prüfen: Es soll sich um eine schwerwiegende Krankheit, die zum Lebensende führt, handeln. Es wurden verschiedene Möglichkeiten der Hilfe und Behandlung erörtert oder ausgeführt, der Patient soll urteilsfähig sein, der Sterbewunsch muss ohne äusseren Druck entstanden und dauerhaft sein.

Die Beurteilung der Urteilsfähigkeit und der Behandlungsmöglichkeiten erfordert grundsätzlich ärztliche, häufig speziell psychiatrische Kompetenz, obwohl auch diese bestritten wird. Zu denken ist hier vor allem an die unterdiagnostizierten Depressionen im Alter, wo der Suizidwunsch ein Krankheitssymptom sein kann [1]. Nun wird die Beurteilung erschwert, wenn die neuen SAMW-Richtlinien aus dem Jahr 2018 berücksichtigt werden. Dort werden neben Krankheitssymptomen neu auch Funktionseinschränkungen, die zu einem unerträglichen Leiden führen, aufgeführt. Weder Funktionseinschränkungen noch unerträgliches Leiden sind näher definiert, was für den Arzt so kaum beurteilbar ist. Diese Ausweitung weg vom todkranken Patienten hin zu nicht näher umschriebenen psychosozialen Faktoren stellt den Arzt und letztlich die ganze Gesellschaft vor neue Herausforderungen.

Bei den steigenden Pflege- und Behandlungskosten im Alter - ein Pflegeplatz in einer entsprechenden Institution kann rasch über 10000 Franken monatlich kosten - stellen sich auch Fragen zur Voraussetzung «ohne äusseren Druck». Alte Menschen sind nicht nur selbstbestimmte Individuen, sondern Teile einer Familie und der Gesellschaft. Begriffe wie «altruistischer Suizid» oder «alterssuizidfördernde Gesellschaft» mögen hier nur als Ansatz für weitere Diskussionen dienen [2]. Ob alle Sterbehilfeorganisationen uneigennützig ihre Tätigkeit ausüben, bleibt dabei dahingestellt. 


\section{Beihilfe zum Suizid - einige Zahlen}

Das Bundesamt für Gesundheit verzeichnete 2014 bei Personen mit Wohnsitz in der Schweiz 742 Fälle von assistiertem Suizid, was 1,2 Prozent aller Todesfälle ausmacht. Gegenüber dem Vorjahr war das eine $\mathrm{Zu}$ nahme von 26 Prozent. Frauen nehmen Sterbehilfe eher in Anspruch als Männer. Die Betroffenen waren zu 94 Prozent über 55-jährig und litten überwiegend an zum Tode führenden Krankheiten. Zusätzlich reisen Personen aus dem Ausland in die Schweiz, um Suizidbeihilfe in Anspruch zu nehmen. Nicht zu vergessen ist die Dunkelziffer, nämlich die indirekten suizidalen Handlungen wie etwa Risikoverhalten oder Unterlassungshandlungen. Der Vergleich der Suizidraten mit der Anzahl assistierter Suizide zeigt, dass die Suizidrate nach 2008 abnimmt, hingegen die Anzahl der assistierten Suizide besonders bei älteren Menschen zunimmt. Das legt nahe, dass eine wachsende Gruppe älterer Menschen, die sonst Suizid begehen würden, die Möglichkeit eines assistierten Suizids in Anspruch nehmen.

\section{Das Bundesamt für Gesundheit verzeichnete} 2014 bei Personen mit Wohnsitz in der Schweiz 742 Fälle von assistiertem Suizid.

Eine sorgfältige epidemiologische Untersuchung [3] aus dem Jahr 2014 zeigt Daten, die Diskussionsbedarf erzeugen. Zwischen 2003 und 2008 wurden alle von den Sterbeorganisationen gemeldeten assistierten Suizide erfasst. In den fünf Jahren der Erfassung starben 1301 Personen durch einen assistierten Suizid. Davon waren 862 Menschen zwischen 65 und 94 Jahre alt, aber ganze 439, also ein Drittel aller Todesfälle, waren jünger als 65. Bei 84 Prozent (N 1093) waren nachweisbare Gründe für einen assistierten Suizid zu finden, aber bei 16 Prozent fand ein assistierter Suizid ohne eruierbaren Grund wie etwa eine entsprechende Diagnose oder ein naher Tod statt. Bei der Erfassung der Diagnosen resp. Krankheiten, die zur Sterbehilfe geführt haben, ergeben sich für die Jahre 1998 bis 2009, dass bei der Sterbehilfe mehrheitlich Krebserkrankungen und an zweiter Stelle neurodegenerative Erkrankungen die Ursache waren. Aber auch hier findet sich eine grosse Gruppe, etwa ein Fünftel aller Fälle, mit anderen nicht bekannten Gründen.

In einer niederländischen Studie (2010) wünschten 60 Prozent der Befragten für sich Sterbehilfe, aber nur 2,7 Prozent aller Sterbenden nahmen diese dann auch in Anspruch. In einer Umfrage der SAMW (2014) befürworteten 78 Prozent der Ärzte die Beihilfe zum Suizid, aber weniger als die Hälfte davon war bereit, eine solche auch durchzuführen.

\section{Einige Diskussionspunkte}

Bis jetzt hat es der Bundesrat abgelehnt, die Sterbehilfe explizit gesetzlich zu regeln. Er beruft sich auf die bekannten strafrechtlichen Vorgaben, nämlich dass Beihilfe zum Suizid straflos ist, sofern sie ohne selbstsüchtige Beweggründe erfolgt (Art. 115 StGB). Tötung auf Verlangen ist hingegen nach Art. 114 StGB strafbar. Da in unserem Land die Sterbehilfe beinahe exklusiv von Organisationen angeboten wird, stellt sich aber die Frage, ob nicht zumindest deren Aktivitäten gesetzlich geregelt werden müssten.

Weitere Diskussionen laufen dahin, ob für einen assistierten Suizid immer eine Organisation Hand bieten muss oder ob nicht auch individuelle Lösungsansätze denkbar wären. Bis anhin wurde die Tätigkeit von Sterbehilfeorganisationen in Alters- und Pflegeheimen sowie in Spitälern und Kliniken abgelehnt. So muss ein sterbewilliger Heimbewohner meistens verlegt werden, damit der assistierte Suizid zur Ausführung kommen kann. Mit der zunehmenden Diskussion um den assistierten Suizid und den Wünschen von Heimbewohnern und Patienten wird zurzeit auf verschiedenen Ebenen diskutiert, ob Sterbeorganisationen nach bestimmten Regeln in den entsprechenden Institutionen zugelassen werden sollen.

Der von Sterbeorganisationen geforderte sogenannte Altersfreitod verursacht kontroverse und zum Tei emotional geladene Auseinandersetzungen. Soll ein Mensch im Alter auch ohne Krankheit und ohne Vermutung auf einen baldigen Tod, aus eigenem Willen, quasi gesund, ein Recht auf einen assistierten Suizid haben? Heisst Selbstbestimmungsrecht auch das Recht auf Sterbehilfe? Genügt «Lebenssattheit», um mit Hilfe aus dem Leben zu scheiden? Die neuen Richtlinien der SAMW zielen in diese Richtung. Wird hier einem allgemeinen Trend entsprochen und will insbesondere die Ärzteschaft eine solche Entwicklung mittragen?

\section{Heisst Selbstbestimmungsrecht auch das Recht auf Sterbehilfe? Genügt "Lebenssattheit», um mit Hilfe aus dem Leben zu scheiden?}

Ein in der Psychiatrie lange umstrittenes Thema stellt das Recht von psychisch kranken Menschen auf einen assistierten Suizid dar. Wird psychisch Kranken generell ein solches Recht verweigert, führt das zu einer Ungleichbehandlung gegenüber körperlich erkrankten Menschen und trägt zur Ausgrenzung und Stigmatisierung bei. Oft bleibt es aber eine Herausforderung, zwischen wohlüberlegtem Suizidwunsch und Suizidwünschen im Rahmen einer psychischen Erkrankung zu unterscheiden. 


\section{Gedanken zum Schluss}

Die Schweiz ist in den umliegenden Ländern wegen ihrer Offenheit für einen assistierten Suizid bekannt. Das heisst aber keineswegs, dass der assistierte Suizid und die damit verknüpften Fragestellungen wie etwa

\section{Bis jetzt nicht erwähnt ist die Frage, wie das Umfeld eines Menschen auf eine Beihilfe zum Suizid reagiert.} und rechtliche Aspekte gelöst und unbestritten wären. Bis jetzt nicht erwähnt ist die Frage, wie das Umfeld eines Menschen auf eine Beihilfe zum Suizid reagiert. Nachgewiesen ist, dass Angehörige nach einem assistierten Suizid eines Familienmitgliedes häufiger an einer Posttraumatischen Belastungsstörung und Depressionen leiden [4]

Das Recht auf ein selbstbestimmtes Lebensende ist unbestritten. Aber beinhaltet unser Dasein nicht genauso so ein Recht auf Fürsorge? Fortschrittlich ist nicht die Gesellschaft, in der der Suizid zur Selbstverständlichkeit wird, sondern jene, die ausreichend Fürsorge, Zuwen-

Korrespondenz: Dr. med. Hans Kurt, Past-presiden Schweizerische Gesellschaft für Psychiatrie und Psychotherapie SGPP Bielstrasse 109 $\mathrm{CH}-4500$ Solothurn kurt[at]solnet.ch dung und Behandlung anbietet ("Der Bund», 11.6.2012). Wie kann eine Antwort auf den Suizidwunsch auch aussehen? Zu erwähnen sind hier Ansätze wie Palliative Care, Hospizbewegung, niederschwellige und aufsuchende Angebote der Geriatrie und Alterspsychiatrie. Und nicht zuletzt: Ohne eine adäquate, genügende die Organisation, die Rahmenbedingungen, ethische

Finanzierung der Pflege und Betreuung chronisch kranker Menschen und der älteren Bevölkerung besteht die Gefahr, dass diese Menschen niemandem mehr zur Last fallen wollen. Stichwörter dazu sind der altruistische Suizid und die den Alterssuizid fördernde Gesellschaft. Oder andersherum gedacht, dass die Gesellschaft ganz froh ist, wenn diese Menschen rechtzeitig aus dem Leben scheiden.

Idealistische Vorstellungen wie der «schöne Tod», «Sterben in Gesundheit», «Mein Ende gehört mir» helfen im Alltag von Patienten, Pflegenden und Ärzten wenig. Die Diskussion zur Beihilfe zum Suizid soll offen bleiben, sie ist notwendig, damit wir nicht Schlagwörtern und Trends folgen, ohne zu wissen, was wir damit bewirken.

\section{Literatur}

1 Minder J, Ajdacic-Gross V, Hepp U. Alterssuizid. Swiss Medical Forum. 2018;18(10):230-5

2 Wedler H. Selbstbestimmtes Sterben: nur eine Utopie? Psychother im Alter. 2013;11:11-26.

3 Steck N, Junker Ch, Maessen M, et al. Suicide assisted by right-todie associations: a population based cohorty study. Intern J Epidem. 2014;1-9.

4 Wagner B, Müller J, Maercker A. Death by request in Switzerland Posttraumtic stress disorder and complicated grief after witnessing assisted suicide. Europ Psychiat. 2012;27(7):542-6.
Positionspapiere

http://www.ipsilon.ch/de/aktuell/news.cfm, Positionspapier Vorstand IPSILON 2017

http://www.sgap-sppa.ch/fachleute/fachpublikationen, Positionspapier, Suizidbeihilfe im Alter SGAP, November 2014

https://www.samw.ch/de.html, Richtlinien Umgang mit Sterben und Tod 2018 